\title{
FLUORINE IN THE DIET AS A FĄCTOR IN THE PREVENTION OF DENTAL CARIES
}

\author{
By Gilbert J. Parfitt, F.D.S., R.C.S., M.R.C.S., L.R.C.P. \\ British Postgraduate Medical Federation, Institute of Dental Surgery
}

Caries can be controlled by the early treatment of the carious lesion, by alteration of conditions upon the tooth surface and by dietary means during the period of tooth formation.

The incidence of dental caries is influenced by a large number of factors. After the teeth have erupted, local conditions on the surface of the teeth have the greatest influence, but tooth structure is of considerable importance. Teeth that have a rough surface texture are more readily attacked by caries than those presenting a smooth surface, ${ }^{1,2}$ and it has been demonstrated by one observer that a rough surface texture of the enamel. is associated with hypocalcification of the deeper layers of the enamel beneath. ${ }^{3,4}$ Both conditions are considered to be the result of metabolic disturbance, usually of dietary origin, during tooth formation.

\section{Effect of Calcium and Phosphorus in Diet}

Tooth structure is sensitive to metabolic disturbance during tooth formation ${ }^{5}$ and has been shown to be affected in animals by dietary means, particularly by alteration of the calcium, phosphorus and vitamin D levels of the diet.

Toverud $^{6}$ carried out a carefully controlled study in Norway and showed that dental caries could be reduced in children by dietary control carried out over the entire period of tooth formation. This necessitated diet control during the ante-natal and post-natal periods up to the time of tooth eruption.

Under the age of five years, the clinical difference between the dental caries incidence of the controlled and uncontrolled groups was remarkable. However, the percentage difference diminished with age, as both groups above a certain age showed a similar rate of caries incidence, but in each age group those who had undergone diet control had less dental caries.

\section{Effect of Fluorine in Diet}

Epidemiological studies demonstrate that the ingestion of a trace of fluorine during tooth formation also affects the tooth structure and lowers the incidence of dental caries. The addition of fluorine to the diet might, therefore, be a possible means of mass caries control.

The first effect of fluorine upon the teeth to be reported was a white mottling or a brown staining and pitting of the enamel surface. ${ }^{7}$ This condition was noticed in a large percentage of teeth of people living in certain well-defined localities. One hundred and forty of such endemic areas were reported in the U.S.A., and Ainsworth, ${ }^{8}$ in this country, reported upon the mottled teeth found in the inhabitants of Maldon in Essex. Many other reports of such areas have come from various parts of the world.

Ainsworth reported that all the cases showing mottling or staining of the teeth came from within three miles of Maldon and that this disfigurement was not seen in individuals born outside the area and moving into Maldon above the age of eight years, the incidence of mottling decreasing rapidly when the move occurred above the age of three years. He noted that the brown stain first appeared on the most prominent part of the erupted tooth and was then followed by staining of the most prominent part of the next tooth to be exposed in the mouth. This suggested an outside influence or origin of the stain, such as atmosphere or water.

The possible causes of such a clinical condition were considered and the most likely was thought to be the presence of some substance in the drinking water. The water of the Maldon district was analysed and the presence of a higher-than-average concentration of fluorine was found.

Similar observations were made in America ${ }^{9}$ and, in a survey of a large number of such areas, no differences other than the fluorine content of the water supply could be discovered.

In I928 Ainsworth also reported a lower dental caries experience in both deciduous and permanent teeth of the Maldon children, although the deciduous teeth showed no evidence of mottling. Black in $1916^{10}$ and McKay in $1929,{ }^{11}$ a year after Ainsworth's report, noted a decreased amount of caries in other endemic areas.

Following these reports, surveys were carried out to ascertain whether a correlation existed 
between the dental caries experience and the fluorine content of drinking water, and to what extent fluorine was present in foods and public water supplies.

\section{Natural Occurrence of Fluorine}

Fluorine is widely distributed in nature, being present in measurable amounts in almost every article of diet ${ }^{12,13}$ and in many water supplies. Surface water contains only a trace of fluorine and most public water supplies utilizing surface water contain less than 0.2 parts per million (p.p.m.) of fluorine. A number of public supplies, however, obtain water from boreholes which may contain an appreciable amount of fluorine should fluorine be present in the water-bearing strata.

Fluorine occurs in rocks as fluorspar and apatite and is present in the phosphatic beds and clays which occur in many parts of England. ${ }^{14}$ In these areas, if the drinking water is obtained from boreholes, some of the supplies contain as much as 6 p.p.m. of fluorine, as in the Maldon district. Public water supplies in England with above 1.0 p.p.m. of fluorine include Chelmsford, Colchester, Maldon, Slough, South Shields and West Hartlepool, but although these and many boreholes in Essex and a few isolated supplies contain appreciable amounts of fluorine, the majority of drinking waters in this country contain less than 0.2 p.p.m

\section{Amount of Fluorine Ingested}

An average mixed diet contains from 0.2 to 0.3 p.p.m. of fluorine, which supplies between 0.2 and $0.5 \mathrm{mg}$. of fluorine per day, ${ }^{12}, 18$ apart from that taken in the drinking water. Some articles of food contain above 1.o p.p.m. of fluorine, notably cereals, flour and some vegetables; fish may contain up to as much as 15 p.p.m.

The average daily water consumption is between 2 and $2 \frac{1}{2}$ pints, but the amount taken by individuals varies considerably. ${ }^{15,16}$ If the fluorine content of the water is 1.0 p.p.m., $1.5 \mathrm{mg}$. of fluorine may be ingested by this means, in addition to that contained in the solid diet. A total amount of $0.8 \mathrm{mg}$. or more of fluorine per day would be ingested by children drinking a water containing 0.2 p.p.m., whereas more than double this amount might be ingested by those living in a high fluorine area where the water contains from 1.0 to 1.5 p.p.m. of fluorine.

\section{Absorption of Fluorine}

Fluorine in the drinking water is absorbed ${ }^{16}$ from the gut more readily than fluorine contained in the food; $80 \%$ of water-borne fluorine is assimilated, ${ }^{17}$ whereas only $60 \%$ of fluorine in food is absorbed, ${ }^{12}$ the remainder being excreted in the faeces. The fluorine which becomes absorbed is excreted in the urine or sweat.

Absorption of fluorine is influenced by the presence of other substances in the gut, in particular calcium, which when present in sufficient amounts considerably decreases its absorption. In this respect it is of interest to note that, during the first years of life, when tooth formation is active, the diet mainly consists of milk, which not only has a low fluorine content (cow's milk containing only 0.07 to 0.22 p.p.m.), but contains a considerable amount of calcium and replaces the consumption of water. A certain percentage of fluorine, however, is even absorbed from calcium fluoride.

Proprietary brands of baby food may contain more fluorine than raw cow's milk, as they are made up with water which, in some districts, has a high fluorine content, but the amount of calcium in the diet is high and is admixed with the fluorine-bearing water.

At this important period, therefore, not only is the fluorine content of the diet low, but absorption is hindered.

\section{Fluorine Balance}

In a study of the fluorine content of the urine of it was found that, in general, the fluorine conten of the urine was similar to that of the drinking water, ${ }^{17}$. slightly above when the content of the drinking water was below 0.3 p.p.m. and slightly below when the water-content was above 1.2 p.p.m., but this did not necessarily indicate a retention of fluorine by the body. McClure reported that the amount of fluorine absorbed from food, in the various groups studied, was remarkably constant.

The fluorine concentration of the blood is also proportional to the fluorine content of the drinking water. ${ }^{18}$ The average fluorine concentration in the blood of a group of individuals was 0.014 p.p.m. when the drinking water contained 1.12 p.p.m. of fluorine. The highest blood concentration found was o.I p.p.m., one member of each group having this level, which suggested that, although the average blood level of fluorine was raised by taking a high fluorine content water, a concentration of 1.12 p.p.m. did not produce excessive blood levels.

Elimination of fluorine from the body is practically complete if the ingestion is not above 4.0 to $5.0 \mathrm{mg}$. per day. ${ }^{12}$ No toxic accumulation takes place below this level, but if water containing between 1.5 to 3.0 p.p.m. of fluorine is ingested during the period of tooth formation, mottling and staining in a certain percentage of the teeth is produced, but no bone lesions occur. 


\section{Toxicity}

Bone lesions have been described in a few members of a community whose water supply contained 6.0 p.p.m. fluorine, ${ }^{19}$ but many other communities drink water containing a higher fluorine content and no bone lesions have been reported amongst them. Several reports suggest that well-nourished individuals are able to take greater amounts of fluorine without showing signs of fluorosis of either teeth or bones. ${ }^{20}$ It is thought that an ample supply of vitamin $\mathrm{C}$ is of particular importance. The individuals in which the bone lesions were described were particularly ill nourished. ${ }^{19}$

Ainsworth reported that the eruption of the teeth was apparently delayed in children residing in Maldon, ${ }^{8}$ and in Colorado Springs ${ }^{21}$ it is stated that the late eruption found in that community might be associated with the high fluorine content of the water supply (2.6 p.p.m.); no alteration in the dates of eruption were observed in communities using supplies containing under 2.0 p.p.m. of fluorine.

The toxic effects of substances such as fluorine depend upon the quantity ingested, the length of exposure, its elimination from the body and the the tissue tolerance. Cryolite $\left(\mathrm{Na}_{3} \mathrm{AlF}_{6}\right)$ workers may absorb up to $30 \mathrm{mg}$. fluorine daily, the concentration in the urine reaching up to 43 p.p.m..$^{12}$ Retention of fluorine occurs in the bones of such individuals. Excessive concentrations of fluorine in the urine may be demonstrated for as long as seven years after cessation of exposure to cryolite dust.

\section{Fluorine in Teeth}

Several investigators have analysed teeth for their fluorine content. ${ }^{5}$ Less fluorine was found in the enamel than in the dentine, but in each tissue the amount was related to the fluorine content of the water supply. ${ }^{22}$ When the fluorine concentration of the drinking water was between 0.2 to 0.3 p.p.m. the enamel was found to contain 0.01 per cent. fluorine and the dentine 0.023 per cent., when the water content was I.O to I.I p.p.m. the enamel contained 0.013 per cent. and the dentine 0.038 per cent., and when the water content was 7.6 p.p.m. the enamel contained 0.06 per cent. and the dentine 0.12 per cent. No more fluorine was found in non-carious than in carious teeth from the same individual. ${ }^{23}$

\section{Epidemiological Studies \\ Reduction in caries incidence}

As early as 1916, Black ${ }^{10}$ observed a lowered caries incidence in areas of endemic fluorosis, and in 1928 Ainsworth $^{7}$ found that only 7.9 per cent. of the permanent teeth of Maldon children were carious, whereas the average caries incidence of the permanent teeth of similar groups of children in other parts of the country was 13.1 per cent. Caries of the deciduous teeth was also reduced, I2.8 per cent. being carious in the Maldon children as compared with the average of 43 per cent. for the whole country. This reduction in dental caries, peculiar to the Maldon district, was attributed to the same cause as that which produced mottling and staining of the teeth.

Dean, in $1938,{ }^{24}$ carried out a dental survey of school children in two towns in the United States and reported that the children of Galesburg, where the water supply contained I.8 p.p.m. of fluorine, had a dental caries incidence of one-third of that of the children of Quincey, where the water supply was fluorine-free. A wider survey was then catried out in 21 cities, 7,000 children being examined in all. ${ }^{25}$ The data showed a remarkably consistent relationship between the fluorine content of the drinking water and the dental caries experience; caries being consistently less with increasing amounts of fluorine in the water supply.

The incidence and severity of mottling of the teeth was also found to be increased with increasing fluorine content of the water, but the appearance of dental fluorosis was not essential to the lessened dental caries experience, as both the mottled and unaffected teeth became equally carious. A full analysis of the water supplies of these communities was carried out, but no correlation was found to exist between the incidence of dental caries and the hardness of the water or the presence in the water of any substance other than fluorine.

A low dental caries experience has been reported from many areas where the fluorine content of the drinking water is high. In this country, Weaver carried out a dental and nutritional survey of the children of North and South Shields, ${ }^{26}$ two towns similar in many respects, but differing in the fluorine content of the drinking water. The South Shields water is drawn from boreholes in magnesium limestone and was reported to contain, on an average, between $\mathrm{I} .2$ and $\mathrm{I} .8$ p.p.m. of fluorine, whereas the fluorine content of North Shields water supply was 0.25 p.p.m. In each town 500 children aged five years and a similar number aged twelve years were examined. The caries experience of both the deciduous and permanent teeth was. found to be between 50 to 60 per cent. lower in South Shields, which is in close agreement with the findings of .Dean for this particular level of fluorine in the drinking water. Weaver found no evidence of delay in the eruption of the teeth nor any inter-relationship between the incidence of caries, mottling of the teeth and 
nutritional condition. Expressing the difference in caries experience, between the two groups, as a percentage might be misleading, and it was suggested that a truer perspective might be given by expressing the lowered caries experience as a delay in the onset of dental caries. He found that at fifteen years of age the children in South Shields had the same average amount of caries as found in the children of North Shields at 12 years of age and that this, therefore, could be expressed as a delay of dental caries of three years.

In a survey carried out six years later, Weaver ${ }^{27}$ found the difference in the dental caries experience of the two groups was not so great as in the first survey and attributed the change to an alteration in diet.

Weaver also examined a number of adults in North and South Shields and, although there was a difference in the dental caries incidence between the two groups, caries increased year by year at a similar rate, the difference being equivalent to a postponement of caries of about five years. $^{28} \mathrm{He}$ also examined children and adults in West Hartlepool, ${ }^{27}$ where the water contained 2.0 p.p.m. of Huorine, and found that each age group showed a lower caries incidence than comparable groups in South Shields where the water supply contained r.6 p.p.m. of fluorine. Although the dental caries was considerably reduced in West Hartlepool, the amount of mottling of the teeth of the adults was severe.

From these surveys it became clear that the margin between beneficial amounts of fluorine in the drinking water and amounts that produce mottling of the enamel is narrow, and there is a limit to the reduction in caries that can be obtained on account of the adverse effects on the teeth following the ingestion of the higher concentrations of fluorine.

Hodge $^{29}$ analysed the data collected by Dean in his survey of 2 I cities and found that, by plotting the incidence of mottling of the teeth and of caries against the fluorine content of the drinking water upon a logarithmic scale, the data fell on two straight lines. The line representing the decrease in caries crossed the line representing the incidence of mottling at, or above, the level of $\mathrm{I}$ p.p.m. of fluorine and this point of intersection might be called the point of minimal caries with minimal mottling of the enamel.

Mottling of the enamel associated with a fluorine content of the drinking water below 1.5 p.p.m. is so mild as to be unnoticed unless the teeth are specifically examined for the condition, and only above this point is the condition unsightly or the incidence high. At the level of I p.p.m. of fluorine there was no indication that the occasional appearance of white flecks on the enamel surface is, in fact, associated with the presence of fluorine in the drinking water.

This analysis, therefore, demonstrated that a beneficial reduction in dental caries could be produced with little or no injury to the enamel.

\section{Ingestion of Fluorine during Tooth Formation}

Investigations have been carried out to find at what age ingestion of fluorine produces an effect upon the teeth. For this purpose an examination was made of -families that had migrated into high-fluorine areas $^{30}$ and of children from communities whose water supply had been changed to a higher fluorine level. ${ }^{31,32}$

From these studies it was found that the most susceptible period was from birth up to the age of six or eight years. During this period ingestion of fluorine in the drinking water could affect the teeth either as a fluorosis or as a beneficial reduction in dental caries.

The extent to which caries was reduced was shown, therefore, to be dependent upon the length of stay and the age at the time or arrival in the high-fluorine area. If the child was resident for only some of these years, certain of the teeth only were affected and reduction in dental caries was not so marked; the teeth affected being those undergoing a particular stage of calcification at the time. The full effect was obtained only by the ingestion of fluorine continuously over the whole period from birth to the time of eruption of the teeth.

Arnold, however, found no change in dental caries experience in a small group of children who for four years had consumed water, the fluorine content of which had been changed from $0 . \mathrm{I}$ to 0.7 p.p.m.

Russell ${ }^{33}$ also reported upon the dental condition of a group of children in the town of Mitchell, whose water supply had been accidentally fluorinated over a period of eighteen months. The survey was carried out between nine and ten years after exposure. During the eighteen months in question the fluorine content was probably maintained at a level of r.o p.p.m. for a period of six months and at 2.8 p.p.m. for five months; Io per cent. of the children showed a mild degree of mottling, but no change in the dental caries experience was observed. The caries rate of the Mitchell and of comparative groups of children rose continuously with age. Had there been any marked inhibitory effect, the caries incidence curves for the Mitchell children would have deviated at certain ages, but they did not, which demonstrated that no alteration in the caries incidence had occurred and no beneficial effect had followed this temporary fluorination of the water supply. 


\section{Ingestion of Fluorine after Tooth Formation}

It is generally reported that a reduction in dental caries is not produced if the ingestion of fluorine occurs above the age of six to eight years. Deatherage ${ }^{34}$ however, reported a slightly lowered caries experience in a group of 286 Servicemen who had lived in high-fluorine areas for varying lengths of time after their teeth had erupted, and this suggested that the benefit is not entirely limited to the pre-eruptive period. A 30 to 40 per cent. reduction in dental caries incidence has been claimed following the topical application of a 2 per cent. aqueous solution of sodium fluoride to the surfaces of the. teeth soon after their eruption.

\section{Reduction in Dental Caries in Adult Population}

Reports of the dental condition of adults who have spent their entire lives in a high-fluorine area show that the reduction in dental caries, so clearly demonstrated in children of school age, is continued to a certain degree into adult life. Arnold $^{32}$ reports that fewer teeth are missing in adults living in Courtland and Bartlett, where the water contains 5 p.p.m. and 8 p.p.m. fluorine, than in similar individuals living in fluorine-free areas, and Weaver demonstrated a difference in the incidence of caries in adults in North and South Shields which was equivalent to a postponement of five years.

A survey in this country of three high- and three similar low-fluorine areas ${ }^{35}$ demonstrated a difference in caries experience of adults to be equivalent to a delay of ten years. A reduction in caries was observed in children under the age of five years. A very considerable reduction in dental caries extending throughout childhood and into adult life appears, therefore, to follow the ingestion of fluorine-bearing water during the period of tooth formation from birth to six or eight years of age.

\section{Fluoridation of Public Water Supplies}

As many public water supplies contain less than 0.25 p.p.m. of fluorine, the possibility of adding fluorides to the water to maintain the level at, or near, r.o p.p.m. has been considered, and in the United States and Canada four experimental schemes have been in operation for three or four years. ${ }^{36}$ Preliminary reports show between 25 per cent. and 45 per cent. reduction of caries in deciduous teeth and between 20 per cent. and 30 per cent. in permanent teeth.

The full effect of introducing fluorine into the water supply cannot be assessed in less than 15 years, by which time children born in the area will have reached an age when a number of per- manent teeth have erupted and have been exposed to the possibility of carious attack.

A number of local authorities in the United States have considered it unnecessary to await the results of these experiments, and 70 cities are already adding fluorides to their water supplies and 100 others are preparing to do so. The various methods and the cost of water fluoridation has been described by Bull. ${ }^{37}$

\section{Administration of Fluoride in Tablets}

Although fluorine is absorbed appreciably less from food than from water, there is some absorption of fluorine from bone meal, cryolite and calcium fluoride in the gut, ${ }^{16}$ and it has been suggested that, in order to administer the necessary amount of fluorine to children during the period of tooth formation, tablets containing a fluoride should be taken by the individual instead of subjecting the entire community to a water containing a high-fluorine content. Strean and Beaudet ${ }^{38}$ investigated the effectiveness of fluoride-containing tablets in reducing dental caries and claimed some success, but other investigators have found no reduction in dental caries following their use. This method of giving fluorine, therefore, is still in the experimental stage, but has the disadvantage that reliance has to be placed on the patient totake the prescribed number of tablets over a considerable length of time.

\section{Laboratory Investigation of Fluorine Content of Teeth}

The clinical evidence that the fluorine present in the dentine and enamel is derived from systemic ingestion during the period of tooth formation is confirmed by animal experiments and by the fact that unerupted teeth show evidence of fluorosis.

Administration of sodium fluoride to rats by injection demonstrated that a high fluorine intake affects the odontoblast cells, the formation of the dentine matrix, and also the calcification of the enamel and dentine. Such effects were considerably greater when the animals were placed on a low calcium, rachitic diet and, in addition, an uncalcified zone appeared in the tooth substance corresponding in position to the line of development at the time of injection. ${ }^{39}$ This particular reaction did not take place when the animals were fed on a normal diet or on a high calcium rachitic diet.

Analyses of enamel and dentine have demonstrated that the teeth of individuals born in highfluorine areas contain considerable amounts of fluorine. Electron microscopy of such teeth show that the etched surface of the dentine and enamel have a finer structure than is normally seen,40 and electron diffraction and X-ray diffraction 
studies show that the fluorine present in the teeth is in the form of a fluorapatite.

\section{Mechanism of Caries Reduction by Fluorine}

There are several possible mechanisms by which the ingestion of fluorine may reduce dental caries. It may interfere with the acid-producing mechanisms of the oral bacteria or increase the resistance of the tooth structure to acid attack, or interfere with proteolysis.

Sodium fluoride affects the metabolism of oral lactobacilli ${ }^{41}$ and inhibits acid production from glucose in concentrations from 1.0 to roo p.p.m. However, the actual number and growth of the lactobacilli is not greatly affected. Salivary lactobacillus counts are found to be only slightly lower in high-fluorine areas. ${ }^{42,43}$

The presence of fluorine ions inhibits cellular oxidation and also inhibits carbohydrate fermentation by inactivation of enolase.44 Fluorine enters into complex compounds with calcium, magnesium, manganese and iron and in concentrations of 100 p.p.m. many of these metals present in the molecules of enzymes or in the substrate become bound to fluorine and a number of enzyme systems necessary to bacterial activity are wholly or partially inhibited.

Enolase is the most sensitive enzyme and the only one likely to be affected by the concentrations of fluorine found in the mouth. It is thought that the presence of fluorine in the oral cavity in concentrations of r.o p.p.m. introduced in food and water is unlikely to have any marked effect on the carious process.

The fluorine content of the saliva is increased by ingestion of fluorine in the drinking water, 4 p.p.m. giving rise to an average salivary level of o.16 p.p.m. of fluorine. ${ }^{45}$ This concentration is not sufficient to interfere with bacterial activity and clinically it was found that the concentration of fluorine in the saliva had no relation to dental caries experience. A concentration of fluorine is found to occur beneath the enamel surface, and Hodge and Sognnaes ${ }^{46}$. suggested that the fluorine bound in combination with enamel may give relatively high local concentrations of fluorine. This might inhibit the bacterial activity or the enzyme processes which are believed to dissolve the protein and calcified material of the tooth substance, but presumably the combined fluorine would have to be released before it could be made available and some destruction of the tooth substance would necessarily have already occurred. Fluorine may combine with the organic material of the tooth substance and this might profoundly affect proteolysis.

A further explanation of the mechanism of the reduction of dental caries is that the tooth substance is rendered less readily soluble in acids by the presence of fluorine. Should dental caries, $\stackrel{\vec{\rho}}{+}$ therefore, be intimately connected with acid des- $\bar{c}$ truction of the tooth substance, the presence of $\frac{}{\sigma}$ the less readily soluble fluorapatite might explain the lowered caries incidence. Clinical findings $\stackrel{\mathbb{Q}}{\AA}$ point to the presence of fluorine in the tooth substance itself as being the important factor and the latter explanation is possibly the more tenable.

\section{Partial Control of Dental Caries by Ingestion of Fluorine}

Fluorine is not present in foods in sufficient amounts to give any beneficial effect from ingestion and the few articles of diet which contain large $\dot{\omega}$ quantities of fluorine cannot be taken to the 6 exclusion of other foods. Addition of fluorine to the common food substances is not practicable as the dosage is not under control, for varying amounts of the food might be consumed by different individuals; nor would the food substance necessarily reach those most likely to derive benefit from it. But the presence of naturallg occurring fluorine in the drinking water in con centrations of 1.0 to I.2 p.p.m. has not only bee demonstrated as harmless but as causing a con? siderable reduction in dental caries. The artificial introduction of fluorine into the drinking water by addition of fluorides, as carried out in so many districts in the United States of America, would appear to be a most valuable measure in the control - of dental caries.

\section{Summary}

The natural occurrence of fluorine, the amounts ingested in food and water, its absorption and excretion is outlined.

Epidemiological studies upon dental caries incidence of communities using water supplies containing various amounts of fluorine are described.

A considerable reduction in dental caries experience with increasing amounts of fluorine of present in the drinking water has been found, $N$ together with a rise in the incidence of mottled teeth, the earliest sign of a fluorosis. The possible mechanisms of the reduction of dental caries by the ingestion of fluorine and of mass control of dental caries are discussed.

\section{REFERENCES}

'MELLANBY, M., and MELLANBY, H. (1948), Brit. Med. $\mathcal{F}$, $4573,409$.

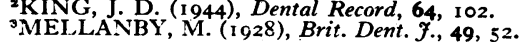

4MELLANBY, M. (1937), Brit. Dent. F., 62, 17.

'LEICESTER, H. M. (1949), ' Biochemistry of the Teeth,' pub. by Henry Kimpton, London.

'TOVERUD, G. (1949), Brit. Dent. F., 86, 191. 
'AINSWOR'TH, N. J. (1928), Roy. Dent. Hosp. Mag., 2, 2-15. ${ }^{8}$ AINSWORTH, N. J. (1933), Brit. Dent. F., 55, 233.

${ }^{9}$ DEAN, T. (1946), 'A.A.A.S. Dehtal Caries and Fluorine,' 5-3 r. ${ }^{10}$ BLACK, G. V., and MCKAY, F. S. (1916), Dental Cosmos, 58, 129. "McKAY, F. S. (1929), Dental Cosmos, 71, 747.

${ }^{12}$ MCCLURE, F. J. (1946), 'A.A.A.S. Dental Caries and Fluorine,' 74-92.

${ }^{13}$ MCCLURE, F. J. (1949), U.S. Pub. Health Repts., 64, 34.

${ }^{14}$ BROMEHEAD, C. N., MURRAY, M. M., et al. (1943), The Lancet, April 17, 1943, 490.

${ }^{1}$ MCCLURE, F. J. (1943), Am. 7. Disease of Children, 66, 362.

${ }^{16}$ MCCLURE, F. J., et al. (1945), 7. Industrial Hyg. and Tox., 27, I59.

${ }^{17 M C C L U R E, ~ F . ~ J ., ~ a n d ~ K I N S E R, ~ C . ~ A . ~(1944), ~ U . S . ~ P u b . ~ H e a l t h ~}$ Rep., 59, 1575.

${ }^{1}$ SMITH, F. A., et al. (1950), 7. Dent. Res., 29, 569.

${ }^{1}$ PANDI'T, C. G., et al. (1940), Indian F. Med. Res., 28, 533.

${ }^{20}$ MURRAY, M. M., and WILSON, D. C. (1948), Rrit. Dent. F., 84, 97.

${ }^{2}$ SHORT, E. M. (1944), F. Dent. Res., 23, 247.

"2:MCCLURE, F. J., and LikiNs, R. C. (1950), F. Dent. Res., 29, 315.

${ }^{23}$ MCCLURE, F. J. (1948); F. Dent. Res., 27, 287.

"IDEAN, H. T., et al. (1939), U.S. Pub. Health Repts., 54, 862.

${ }^{25}$ DEAN, H. T. (1947), Am. f. Orth. and Oral. Surg., 33, 49.

${ }^{26}$ WEAVER, R. (1944), Brit. Dent. F., 75, 29.
${ }^{27}$ WEAVER, R. (1950), Brit. Dent. F., 88, 23 I.

${ }^{28}$ WEAVER, R. (1948), Proc. Roy. Soc. Med., 41, 284.

${ }^{29}$ HODGE, H. D. (1950), F. Am. Dent. Ass., 40, 436 .

${ }^{30}$ KLEIN, H. (1947), Science, 105, 45.

${ }^{31}$ KLEIN, H. (1948), U.S. Pub. Health Rept., 63, 563.

${ }^{32}$ ARNOLD, F. A., JUN. (1946), 'A.A.A.S. I)ntal Caries and Fluorine,' 99-107.

${ }^{33}$ RUSSELL, A. L. (1949), f. Dent. Res., 28, 298.

${ }^{34}$ DEATHERAGE, C. F. (1943), $\mathcal{Y}$. Dent. Res., $22,129$.

${ }^{3}$ FORREST, J. R., PARFITT, G. J., et al., in press.

${ }^{36}$ BULl, F. A. (1950), F. Am. Dent. Ass., 41, 146.

37BULL, F. A. (1951), Ұ. Am. Dental Ass., 42, 29.

${ }^{38}$ STREAN, L. P., and BEAUDET, J. P. (1945), N. Y. State F. of Med., 45,2183 .

$3^{9}$ IRVING, J. 'T. (1949), f. Dent. Res., 28, i 7.

${ }^{\circ}$ GEROULD, C. H. (1945), F. Dent. Res., 24, 22.

${ }^{4}$ CLAPPER, W. E. (1947), Proc. Soc. Exp. Bio. and Med., 65, 333. 42DEAN, H. T., et al. (1941), U.S. Pub. Health Rept., 56, 365.

${ }^{4}$ ARNOLD, F. A., DEAN, H. T., et al., U.S. Pub. Health Repts., 57,773 .

"BOREI, H. (1945), 'Arkiv. for Kemi-Mineraloci och geoloci 20A,' N. $8,208$.

${ }^{45}$ MCCLURE, F. J. (I94I), Amer. F. Dis. Child., 62, 5 I 2.

${ }^{46}$ HODGE, H. C., and SOGYNAES, R. F. (1946), A.A.A.S. Dental Caries and Fluorine,' 53-7.3.

\title{
WOMEN IN MEDICINE: THE EARLY YEARS
}

\author{
By MaY Thorne, O.B.E., M.D., F.R.C.S.I., L.S.A.
}

A hundred years ago a stirring began to be felt by a few women both here and in America that they should be able to prepare themselves to enter the medical profession. Elizabeth Blackwell was the first woman in America who, about $185^{\circ}$, made up her mind to study medicine. She had no idea at that time of the difficulties that stood between her and qualification, but by dint of attending a few lectures here and there, by making use of every scrap of experience, by reading and by the help of a few physicians who sympathized with her in her ambition, she, after some years of hard struggle and disappointing work, managed to present herself for examination at the University of Geneva in the State of New York, passed and became a qualified physician.

In England a few years later Elizabeth Garrett had similar ambitions. She met with similar trying experiences, but she, too, persevered in an indomitable way. She had the friendship of some of the leading medical men, who gave her sym- pathy, though they could give her little help; but she got a little teaching and experience here and there and made the best possible use of it. After some years she learned that the Society of Apothecaries of London admitted, to their licence to practise medicine, students who held the certificate of a practical apothecary. She became apprenticed and subsequently qualified as an apothecary, and in 1865 applied for admission to present herself at the final examination. She was admitted to the examination, passed and was thus the first woman in England legally qualified to practise as a physician of medicine. The Society was apparently horrified at what it had done, and at once took steps to alter its regulations for admission to the examination, by making a rule that in future, certificates of instruction from a recognized medical school must be presented by all candidates for the final examination. This regulation prevented any other woman from presenting herself for the final examination. 